\title{
Correction to: Endogenous oligodendroglial alpha-synuclein and TPPP/p25a orchestrate alpha-synuclein pathology in experimental multiple system atrophy models
}

\author{
Panagiota Mavroeidi ${ }^{1} \cdot$ Fedra Arvanitaki $^{1}$ - Anastasia-Kiriaki Karakitsou ${ }^{1} \cdot$ Maria Vetsi $^{1} \cdot$ Ismini Kloukina ${ }^{2}$. \\ Markus Zweckstetter ${ }^{3,4} \cdot$ Karin Giller $^{4} \cdot$ Stefan Becker ${ }^{4}$. Zachary A. Sorrentino ${ }^{5,6}$. Benoit I. Giasson ${ }^{5,6,7}$. \\ Poul Henning Jensen ${ }^{8} \cdot$ Leonidas Stefanis $^{1,9} \cdot$ Maria Xilouri $^{1} \mathbb{}$
}

Published online: 3 September 2019

(c) Springer-Verlag GmbH Germany, part of Springer Nature 2019

\section{Correction to: Acta Neuropathologica (2019) 138:415-441 https://doi.org/10.1007/s00401-019-02014-y}

The original version of this article unfortunately contained a mistake. The following text was missing in the acknowledgements section.

"This research is co-financed by Greece and the European Union (European Social Fund-ESF) through the Operational Programme "Human Resources Development, Education and Lifelong Learning" in the context of the project "Strengthening Human Resources Research Potential via Doctorate Research" (MIS-5000432), implemented by the State Scholarships Foundation (IKY)."

The correct acknowledgements section should be:

Acknowledgements The authors would like to thank the BRFAA biological imaging facility for their valuable contribution to confocal imaging and image analysis, George Tzanoukos (M.Sc., Head of the

The original article can be found online at https://doi.org/10.1007/ s00401-019-02014-y.

Maria Xilouri

mxilouri@bioacademy.gr

1 Center of Clinical Research, Experimental Surgery and Translational Research, Biomedical Research Foundation of the Academy of Athens (BRFAA), 4 Soranou Efesiou Street, 11527 Athens, Greece

2 Center of Basic Research, Biomedical Research Foundation of the Academy of Athens, Athens, Greece

3 German Center for Neurodegenerative Diseases (DZNE), Von-Siebold-Str. 3a, 37075 Göttingen, Germany

4 Department for NMR-based Structural Biology, Max Planck Institute for Biophysical Chemistry, Am Faßberg 11, 37077 Göttingen, Germany
Biomedical Engineering Department, BRFAA) for technical assistance, and the BRFAA Animal Facility. The authors would also like to thank Dr. Nadia Stefanova (Innsbruck University) for providing the C57BL6 PLP-haSyn transgenic mice and Imago Pharmaceuticals for providing the 11A5 antibody. The authors also like to thank Dr. Lee Clough for editing the manuscript. PHJ was supported by the Lundbeck foundation Grant R223-2015-4222 and R248-2016-25. This work was supported by an MSA Coalition grant and a Bodossaki Grant to MX. Partial support was provided by an NIH grant NS1000876 to BG and by the Innovative Medicines Initiative 2 Joint Undertaking under Grant agreement No. 116060 (IMPRiND) to LS. This Joint Undertaking receives support from the European Union's Horizon 2020 research and innovation program and EFPIA. This work was supported by the Swiss State Secretariat for Education, Research and Innovation (SERI) under contract number 17.00038. The opinions expressed and arguments employed herein do not necessarily reflect the official views of these funding bodies. This research is co-financed by Greece and the European Union (European Social Fund-ESF) through the Operational Programme "Human Resources Development, Education and Lifelong Learning" in the context of the project "Strengthening Human Resources Research Potential via Doctorate Research" (MIS-5000432), implemented by the State Scholarships Foundation (IKY).

Publisher's Note Springer Nature remains neutral with regard to jurisdictional claims in published maps and institutional affiliations.

5 Department of Neuroscience, University of Florida, Gainesville, FL 32610, USA

6 Center for Translational Research in Neurodegenerative Disease, University of Florida, Gainesville, FL 32610, USA

7 McKnight Brain Institute, University of Florida, Gainesville, FL 32610, USA

8 DANDRITE-Danish Research Institute of Translational Neuroscience and Department of Biomedicine, University of Aarhus, Aarhus C, Denmark

9 1st Department of Neurology, Eginition Hospital, Medical School, National and Kapodistrian University of Athens, Athens, Greece 